\title{
Large hiatal hernia: minimizing early and long-term complications after minimally invasive repair
}

\author{
Elettra Ugliono, Fabrizio Rebecchi, Elisabetta Seno, Mario Morino \\ Department of Surgical Sciences, University of Torino, Torino 10126, Italy.
}

Correspondence to: Prof. Fabrizio Rebecchi, Department of Surgical Sciences, University of Torino, Corso A.M. Dogliotti 14, Torino 10126, Italy. E-mail: Fabrizio.rebecchi@unito.it

\begin{abstract}
How to cite this article: Ugliono E, Rebecchi F, Seno E, Morino M. Large hiatal hernia: minimizing early and long-term complications after minimally invasive repair. Mini-invasive Surg 2021;5:2. http://dx.doi.org/10.20517/2574-1225.2020.93
\end{abstract}

Received: 28 Sep 2020 First Decision: 13 Nov 2020 Revised: 29 Nov 2020 Accepted: 9 Dec 2020 Published: 7 Jan 2021

Academic Editor: Uberto Romario Fumagalli Copy Editor: Miao Zhang Production Editor: Jing Yu

\begin{abstract}
Paraesophageal Hernia (PEH) is the protrusion of the stomach and/or other abdominal viscera into the mediastinum due to an enlargement of the diaphragmatic hiatus. The treatment of PEH is challenging: On the one hand, watchful waiting carries the risk of developing acute life-threatening complications requiring an emergency operation. On the other hand, elective repair of PEH has non-negligible morbidity and mortality rates, also due to the characteristics of $\mathrm{PEH}$ affected patients, who are generally elder and frail. A review of the literature is presented to highlight strategies that can be adopted to minimize early and long-term complications after PEH surgical repair. The laparoscopic approach has been shown to provide reduced hospital stay, postoperative morbidity and mortality, and overall costs compared to traditional open surgery, and it is currently considered the standard approach both to elective and emergency operations. The evidence suggests that strict adherence to surgical principles, such as hernia sac excision, extended mediastinal dissection of the esophagus, and tension-free crural repair with or without mesh are mandatory to achieve optimal surgical outcomes and reduce PEH recurrence rate. Different shapes, materials, and techniques of prosthetic repair and the use of relaxing incisions have been proposed, but long-term data are lacking, and no conclusions can be drawn regarding the ideal method of crural closure. When a short esophagus is recognized despite extensive mediastinal dissection, esophageal lengthening procedures are indicated. Systematic addition of a fundoplication is strongly encouraged, for either treating gastroesophageal reflux or reducing recurrence rate.
\end{abstract}

Keywords: Hiatal hernia, paraesophageal hiatal hernia, fundoplication, complications

\footnotetext{
(ㄷ) $\left(\frac{1}{\infty}\right.$

(C) The Author(s) 2021. Open Access This article is licensed under a Creative Commons Attribution 4.0 International License (https://creativecommons.org/licenses/by/4.0/), which permits unrestricted use, sharing, adaptation, distribution and reproduction in any medium or format, for any purpose, even commercially, as long as you give appropriate credit to the original author(s) and the source, provide a link to the Creative Commons license, and indicate if changes were made.
}

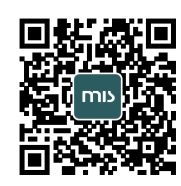




\section{INTRODUCTION}

Hiatal hernia $(\mathrm{HH})$ is the protrusion of an abdominal organ into the mediastinum through the diaphragmatic hiatus.

There are four main types of HH: Type 1 ("sliding"), the most common, is the herniation of the esophagogastric junction (EGJ) above the diaphragm, leaving the stomach in the abdomen; Type 2 ("pure paraesophageal") is the thoracic migration of the gastric fundus while the EGJ remains in the correct position; Type 3 ("mixed") is a combination of both Type 1 and Type 2 components; and, in Type 4 ("giant") $\mathrm{HH}$, the herniation involves the entire stomach along with other abdominal viscera, including colon, omentum, small bowel, liver and spleen ${ }^{[1]}$. Types 2-4 HH are defined as paraesophageal hernias (PEH) and share the same preoperative work-up and surgical treatment ${ }^{[2]}$.

Clinical manifestations of PEH include obstructive (dysphagia and postprandial fullness) and compressive (respiratory complications and recurrent pneumonia) symptoms, gastroesophageal reflux (GER) (heartburn and regurgitation), and chronic anemia. PEH can also present acutely with complications: bleeding, acute obstruction, and strangulation resulting in gastric necrosis ${ }^{[3]}$.

The diagnosis is made with upper endoscopy and barium esophagogram, to assess the morphology of HH. Other examinations, such as computed tomography scan and esophageal manometry, could be helpful in treatment planning, but they are not mandatory ${ }^{[1,4]}$.

\section{INDICATIONS FOR SURGERY}

\section{Elective vs. emergent}

In contrast to Type 1 sliding $\mathrm{HH}$, which does not require surgical intervention unless in the presence of severe GER, PEH carries the potential for severe acute complications ${ }^{[5]}$.

In the past, $\mathrm{PEH}$ repair was proposed for all surgically fit patients, regardless of symptoms, due to previous studies demonstrating an unacceptably high mortality rate (ranging 29\%-56\%), associated with acute presentations $^{[6,7]}$.

A study from Stylopoulos et al. ${ }^{[8]}$ changed this paradigm. The authors performed a Markov Monte Carlo decision analysis to address the optimal treatment strategy for PEH. The input variables considered, obtained from a systematic review of the literature and data of the 1997 Nationwide Inpatient Sample, were: the estimated mortality rate after elective laparoscopic (1.4\%, range $0 \%-5.2 \%)$ and emergency (5.4\%) PEH repair, the annual probability of developing symptoms progression (13.8\% range $8.1 \%-21.7 \%$ ), the annual probability of acute presentation requiring emergency surgery of untreated patients ( $1.1 \%$ range $0.7 \%-1.9 \%$ ), and the annual probability of $\mathrm{HH}$ recurrence after surgical repair (1.9\% range $0.3 \%-5.4 \%)$. With these assumptions, the authors estimated that watchful waiting would be the optimal treatment for $83 \%$ of $\mathrm{PEH}$ patients, as the risk of developing life-threatening complications is only $1.1 \%$ per year.

Since then, other studies have demonstrated lower mortality rates associated with PEH repair, both in the elective and in the emergency setting ${ }^{[9,10]}$. Even with these new reports, an updated study using the same statistical methodology achieved the same conclusions in terms of mortality ${ }^{[11]}$. However, considering costeffectiveness, a similar study performed by Morrow et al. ${ }^{[12]}$ concluded that elective repair, although more expensive, guarantees superior quality of life compared to watchful waiting. Current guidelines recommend the elective repair of all symptomatic $\mathrm{PEH}$, while in asymptomatic patients the indications to elective surgery must be balanced with the patient's age and comorbidities ${ }^{[5]}$. 


\section{Open vs. minimally invasive approach}

The conventional open approach to PEH repair, through a thoracotomy or a laparotomy, was associated with a high rate of morbidity (5.3\%-25\%) and mortality (0\%-3.7\%). The main complications described were pneumonia $(2.6 \%$, range $2.1 \%-8.7 \%)$ and wound infections $(5.8 \% \text {, range } 0.8 \%-8.7 \%)^{[13]}$. Since the introduction of the laparoscopic technique to PEH treatment by Cuschieri ${ }^{[14]}$ in 1992, the minimally invasive approach has spread rapidly. Several population-based studies demonstrated a significant reduction in hospital stay, intensive care unit stay, postoperative morbidity, mortality, and overall costs of laparoscopic PEH repair compared to the conventional open approach ${ }^{[15,16]}$. Therefore, laparoscopy is considered the preferred surgical access for PEH repair, including in the emergency setting ${ }^{[5,17]}$.

More recently, the robotic platform has been proposed for surgical PEH treatment. The evidence regarding robot-assisted repair of $\mathrm{PEH}$ consists of small retrospective series of single institutions in their early experience with this technique, and no long-term follow-up is available. These studies described a postoperative morbidity of $15 \%-23 \%$ and a mortality rate of $0 \%-2.5 \%$, which are comparable with the outcomes of the laparoscopic series reported in the literature ${ }^{[18-20]}$.

However, no studies specifically assessing the comparison of robot-assisted and laparoscopic approaches to $\mathrm{PEH}$ repair have been conducted, and no clear benefits of the robotic approach have been elucidated yet. Therefore, the role of robotics in the surgical management of PEH remains controversial.

\section{SURGICAL PRINCIPLES}

The essential technical steps of the procedure consist of complete reduction of $\mathrm{HH}$, hernia sac excision, extensive mediastinal mobilization of the esophagus, and tension-free crural closure.

The first step of the procedure is the abdominal reduction of $\mathrm{HH}$ contents by gentle traction of the hernia sac, proceeding gradually with extensive mediastinal mobilization of the esophagus with blunt dissection in order to obtain at least $2-2.5 \mathrm{~cm}$ of intra-abdominal esophageal length [Figure $1 \mathrm{~A}$ and $\mathrm{B}]^{[21]}$.

During hernia sac dissection, caution must be used to prevent injury to the vagal nerves on the anterior and posterior aspect of the esophagus, to the pleura, and to the adjacent vascular structures [Figure 2] ${ }^{[22]}$.

After complete reduction, sac excision is imperative [Figure 3]. A tension-free closure of the diaphragmatic crura must be achieved with crural approximation with or without mesh [Figure 4A and B]. Additional technical steps, such as fundoplication, esophageal lengthening, gastropexy, and relaxing incisions, have been investigated to improve the results of PEH repair and are discussed below.

The most common intraoperative complication reported is visceral injury (esophageal and gastric perforations), which is reported in up to $11 \%$ of cases, followed by vagal nerve injury and pulmonary complications (pneumonia) ${ }^{[23]}$.

Sudden increases in intra-abdominal pressure in the immediate postoperative period, due to coughing, belching, vomiting, and lifting weights, have been shown to contribute to $\mathrm{PEH}$ recurrence ${ }^{[24]}$. Therefore, postoperative nausea and vomiting must be treated aggressively ${ }^{[5]}$.

Routine early upper gastrointestinal series before starting diet is unhelpful in the absence of suspicious clinical signs, as it has been shown that it would change the clinical management of patients in only $0.8 \%$ of cases $^{[25]}$. 

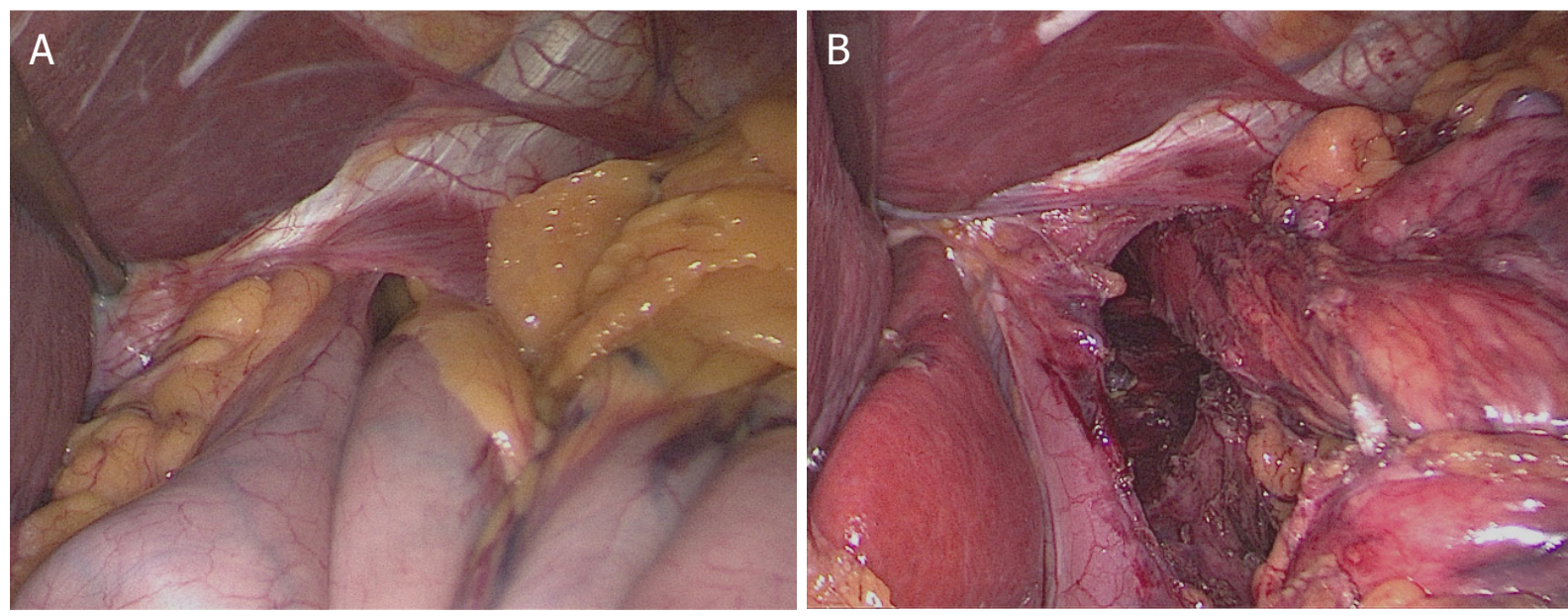

Figure 1. Hernia content reduction: (A) reduction of hiatal hernia contents by gentle traction of the hernia sac; and (B) obtaining at least $2-2.5 \mathrm{~cm}$ of intra-abdominal esophageal length

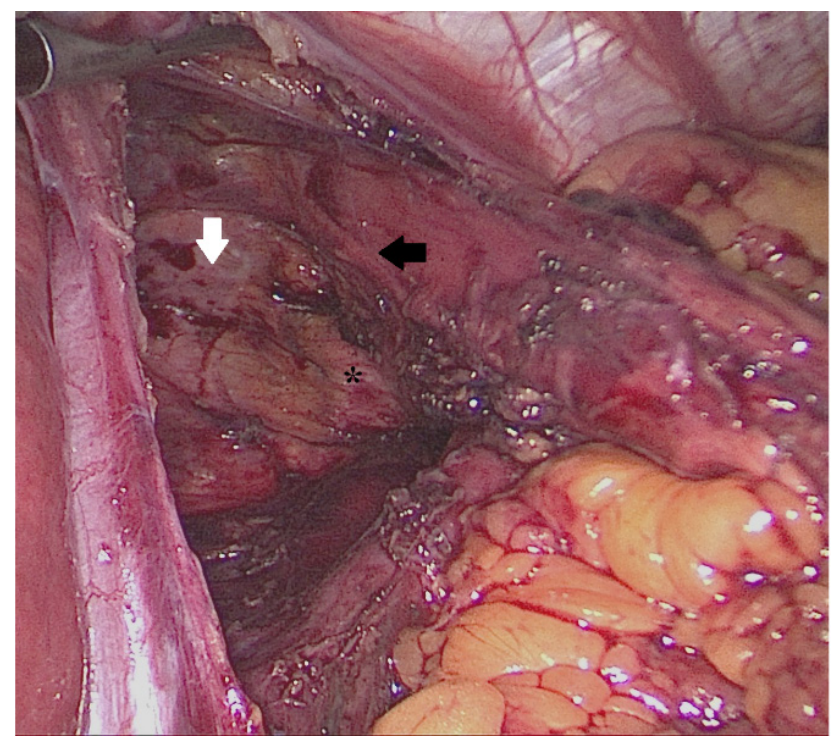

Figure 2. During hernia sac dissection, caution must be used to prevent injury to the vagal nerves on the anterior and posterior aspect of the esophagus, to the pleura, and to the adjacent vascular structures. White arrow, pleura; black arrow, posterior vagus nerve; asterisk, aorta

\section{POSTOPERATIVE COMPLICATIONS}

\section{PEH recurrence}

A significant rate of recurrences after PEH repair has been reported, although patients are often asymptomatic ${ }^{[26]}$. "Radiological" recurrences are described in up to $20 \%-30 \%$ of cases, while only $5 \%$ of patients would require surgical revision ${ }^{[27]}$.

Several technical factors have been investigated in an attempt to reduce the rate of PEH recurrences: PEH sac excision, the method of crural closure, the addition of an esophageal lengthening procedure, and the addition of a gastropexy.

\section{PEH sac excision}

To reduce the risk of recurrence, complete excision of the hernia sac should be performed whenever feasible ${ }^{[28]}$. This fundamental step of the procedure accomplishes several objectives: first, it represents 


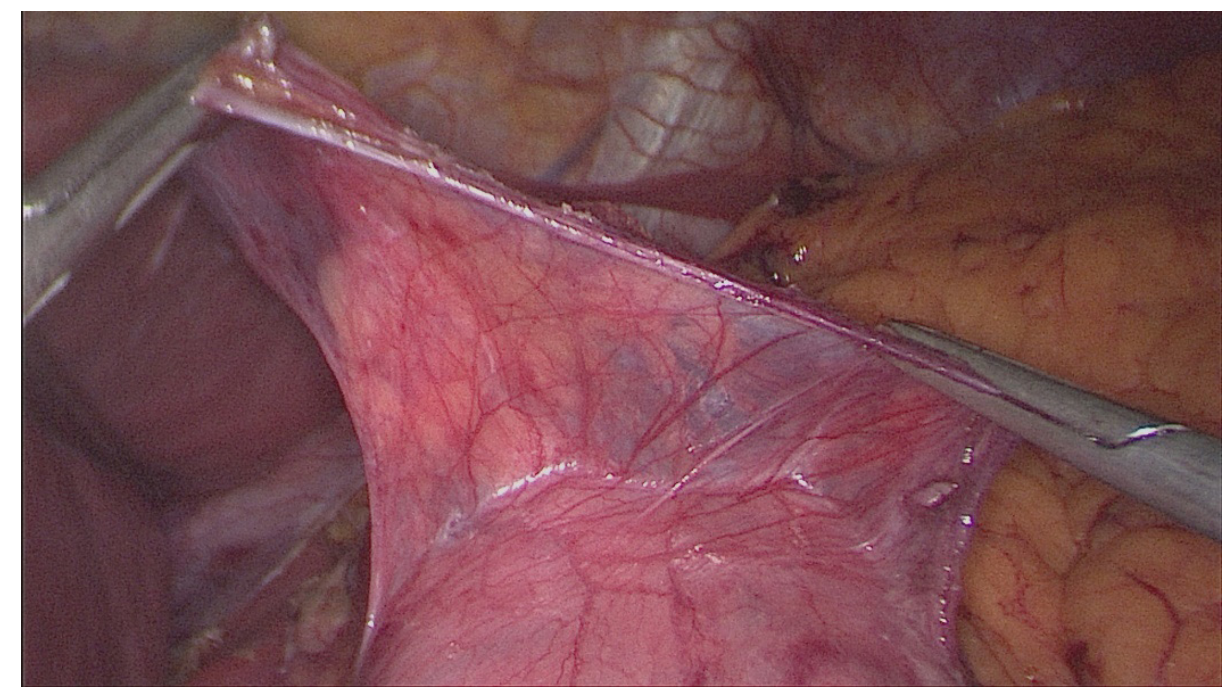

Figure 3. Identification of the hernia sac
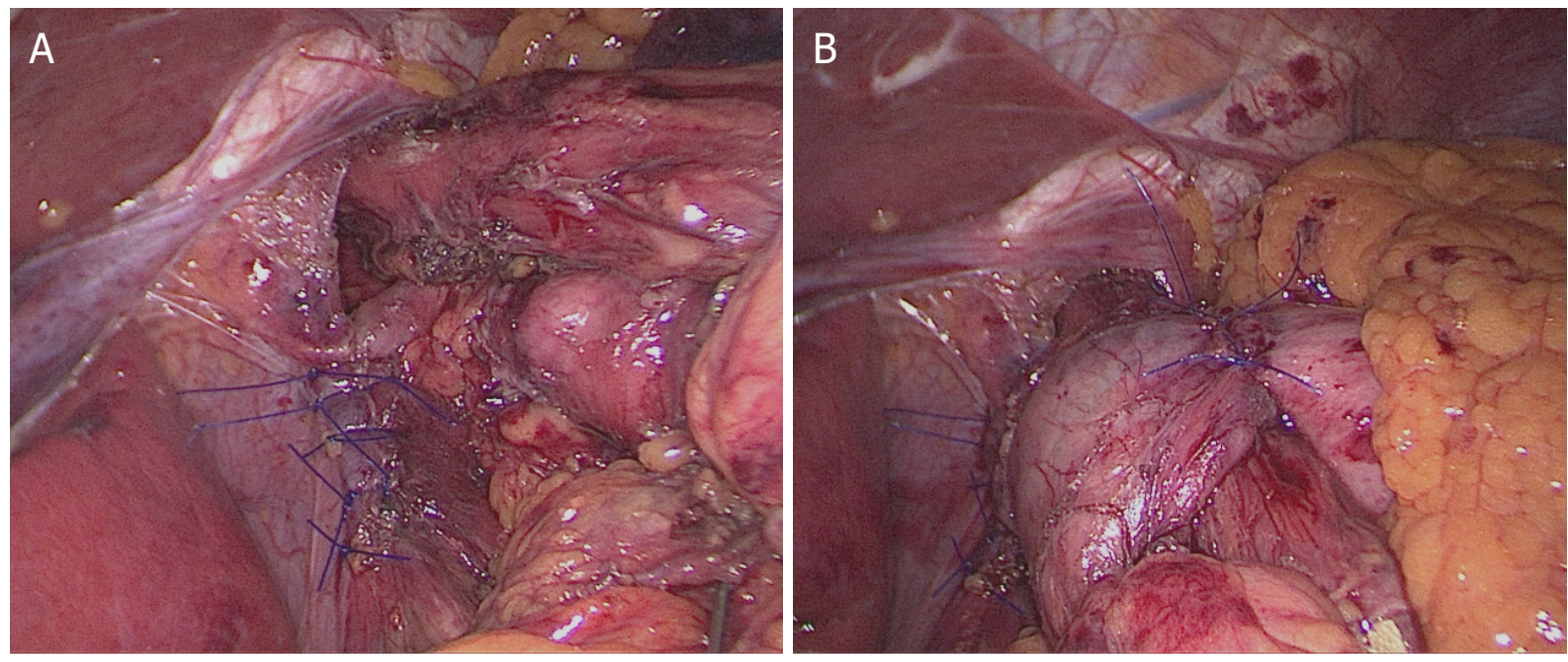

Figure 4. Paraesophageal hernia repair: (A) cruroplasty; and (B) total $360^{\circ}$ fundoplication

the correct plane of dissection, avoiding potential injuries to the neural and vascular adjacent structures; second, it reduces the risk of collections in the thoracic cavity; and third, since the hernia sac acts as a lead point that pushes the stomach back in the thoracic cavity, its excision reduces the risk of $\mathrm{HH}$ recurrence ${ }^{[29]}$.

\section{Crural closure: mesh vs. simple cruroplasty}

Closure of the diaphragmatic hiatus is mandatory during PEH repair. It can be achieved through several techniques, with primary closure or the use of a mesh. The prosthetic materials can be used as a reinforcement of a primary crural closure or as a "bridge" to close a wide diaphragmatic defect without any attempt to approximate the crural pillars. Moreover, some authors suggest performing crural relaxing incisions to achieve a tension-free crural closure ${ }^{[30]}$.

In the early laparoscopic series, simple primary cruroplasty was associated with an unacceptably high rate of recurrences at medium follow-up, described in up to $42 \%$ of patients ${ }^{[31]}$. 
In light of the good results achieved with the introduction of prosthetic materials in inguinal and ventral repair surgery, the use of meshes has been proposed also in PEH repair. There is a wide array of configurations, materials (including synthetic non-absorbable, absorbable, or biologic matrices), and methods of fixation of the mesh (anterior, posterior, or circumferential, with staples, tacks, sutures, or glue) ${ }^{[32-36]}$.

Several studies showed a reduced recurrence rate with the use of synthetic meshes. For instance, Frantzides et al. ${ }^{[37]}$ performed in 2002 a randomized controlled trial (RCT) of patients undergoing laparoscopic PEH repair with simple (36 patients) vs. reinforced polytetrafluoroethylene (PTFE) cruroplasty (36 patients). The recurrence rate, verified with barium contrast studies, was significantly higher in the simple cruroplasty group compared with the PTFE group $(22 \% v s .0 \%, P<0.006)$.

Disadvantages related to the use of synthetic materials include the risk of mesh adhesion, erosion of the esophageal wall, and extensive fibrosis resulting in the onset of troublesome dysphagia ${ }^{[38]}$.

Biological and absorbable meshes have been proposed to overcome the downsides of synthetic meshes. Oelschlager et al ${ }^{[39]}$ performed a multicenter RCT to test the efficacy of crural reinforcement with a biological mesh derived from porcine small intestinal submucosa (51 patients) compared to primary crural closure (57 patients). The authors published in 2006 the phase 1 results of the trial, showing a significant reduction in radiological PEH recurrences compared to primary repair (9\% vs. 24\%) at six-month followup. However, a longer follow-up of the same study showed a high rate of recurrences, with no significant differences between the two groups (59\% in the mesh group $v s .54 \%$ in the primary repair group ${ }^{[40]}$.

The short-term results of biological meshes were also confirmed in a systematic review and metaanalysis performed by Antoniou et al ${ }^{[41]}$ including five studies comparing simple suture $v$ s. biologic mesh cruroplasty. However, no long-term data were available for analysis.

Watson et al. ${ }^{[42]}$ performed a multicenter RCT in 2015 with the aim of comparing three methods of $\mathrm{PEH}$ repair: primary suture (43 patients), absorbable mesh (41 patients), and non-absorbable mesh (42 patients) cruroplasty. A combined radiological and endoscopic assessment of recurrences was performed at 12-month follow-up, and no significant difference was found among the three groups. These results were also confirmed at five-year follow-up ${ }^{[43]}$.

Several meta-analyses described a significant reduction in the recurrence rate at medium-term follow-up, including a lower risk of surgical revision, with the use of prosthetic materials, but the quality of analyzed data was poor and therefore the results are of limited level of evidence ${ }^{[4,45]}$. For instance, Tam et al. ${ }^{[46]}$ performed in 2016 a systematic review and meta-analysis of studies assessing the comparison between primary repair and the use of synthetic mesh. They reviewed 13 publications including RCTs and observational studies. The overall recurrence rate was found to be $24 \%$ (91/382) for the suture group compared to $13 \%$ (46/354) for the mesh group. However, follow-up was significantly shorter, with only half of the patients available for follow-up in the mesh group, therefore recurrences could be underestimated. The authors concluded that the available evidence is of low quality and high risk of bias and does not allow drawing definitive conclusions.

Furthermore, more recent series comparing primary $v s$. mesh reinforced cruroplasty have shown similar outcomes in terms of recurrences at long-term follow-up ${ }^{[47,48]}$. For instance, Koetje et al. ${ }^{[49]}$ reported the comparison between primary repair (127 patients) and mesh reinforced (62 patients) cruroplasty with a follow-up of 40 months. The overall rate of radiological recurrence was similar between the two groups (25.8\% mesh vs. $23.6 \%$ no mesh), with similar reoperation and symptomatic recurrence rates. 
To date, there is no high-level objective evidence recommending the use of meshes in PEH surgical treatment, nor demonstrating the superiority of a specific technique over another. The ideal mesh does not exist, and the choice of the technique largely depends on the surgeon's preferences ${ }^{[50,51]}$. Current guidelines admit that no recommendations can be made regarding the use of mesh in PEH repair ${ }^{[5]}$.

\section{"Short esophagus" and esophageal lengthening}

The entity of the "short esophagus" (SE) is debated. SE is defined as less than $2-2.5 \mathrm{~cm}$ of intra-abdominal esophageal length after extensive mediastinal dissection ${ }^{\left[5_{2}\right]}$. The estimated incidence of the SE is reported to be $1.9 \%-20 \%$ and is thought to be caused by fibrosis and scarring of chronic severe GER insult ${ }^{[4]}$. Some authors question the real existence of SE, claiming the presence of "apparent" SE: a normal-length esophagus that is folded into the chest and appears to be short before extensive mediastinal mobilization ${ }^{[53]}$. The use of routine intraoperative endoscopy during PEH repair is suggested to detect $\mathrm{SE}^{[54]}$.

When a "real" SE is recognized intraoperatively, esophageal lengthening procedures, such as Collis-Nissen fundoplication, are indicated ${ }^{[55]}$. The current technique consists of a totally laparoscopic gastroplasty, performed with a circular stapler, to create a trans-gastric window, through which a linear stapler is introduced to create the "neo-esophagus" ${ }^{\text {"56] }}$. The results of this procedure, performed with the laparoscopic approach, are similar to those reported with the open technique, with a recurrence rate of $25-13 \%{ }^{[4]}$.

However, Collis-Nissen fundoplication is a challenging procedure, with a reported morbidity rate of 19\%$36 \%$, including atelectasis, pneumonia, pneumothorax, and pleural effusion ${ }^{[57]}$. Moreover, it carries a higher risk of leak compared to fundoplication alone $(2.7 \% v s .0 .6 \%)^{[58]}$.

\section{Anterior gastropexy}

Anterior gastropexy was first described by Boerema in 1969, but it was abandoned due to a reported excessively high risk of recurrence, which occurred in $60 \%$ of patients ${ }^{[59,60]}$. With the recognition of the importance of the fundamental technical steps of the procedure, such as sac dissection and excision, that were not performed at the time of the original Boerema procedure, this technique has been modified and proposed again. To date, there are limited data regarding the role of anterior gastropexy, in particular without associated procedures such as mesh cruroplasty or fundoplication, in PEH surgical treatment [Table 1]. Only Daigle et al ${ }^{[68]}$ performed a multicenter study of $101 \mathrm{PEH}$ repair with anterior gastropexy without fundoplication, showing an acceptable recurrence rate of $16.8 \%$ at 12 -month follow-up and avoiding complications of mesh positioning and anti-reflux procedures. However, $29.7 \%$ of patients experienced some degree of postoperative GER.

More recently, several authors have described the use of this procedure in the acute setting or in high-risk patient ${ }^{[6,70]}$. In these situations, the procedure was considered attractive because it does not require long operative times or advanced technical skills even with the minimally invasive approach, and does not affect the possibility to perform subsequent elective $\mathrm{PEH}$ repair.

For instance, Yates et al. ${ }^{[69]}$ reported the results of 11 high operative risk patients presented with acute gastric volvulus and treated with laparoscopic anterior gastropexy. There were no intraoperative complications, but two patients required reintervention. The authors concluded that laparoscopic anterior gastropexy could be considered a valid surgical alternative for frail patients.

\section{Gastroesophageal reflux}

The systematic or tailored addition of a fundoplication during PEH repair is a matter of debate. 
Table 1. Outcomes of laparoscopic gastropexy in paraesophageal hernia treatment

\begin{tabular}{|c|c|c|c|c|c|c|c|c|}
\hline Authors & Year & $n$ & GP (n) & $\begin{array}{c}\text { Associated } \\
\text { procedures }(n)\end{array}$ & Recurrences (\%) & Mortality (\%) & $\begin{array}{l}\text { Follow-up } \\
\text { (months) }\end{array}$ & Notes \\
\hline Agwunobi et al. ${ }^{[61]}$ & 1998 & $13 \mathrm{HR}$ & 13 & & $14.4 \%$ symptomatic & 7.7 & 10 & $15.4 \%$ conversions \\
\hline Hawasli et al. ${ }^{[62]}$ & 1998 & 27 & 25 & $M C=25$ & $0 \%$ & 0 & $1-56$ & $22.2 \%$ reflux \\
\hline Van der Peet et al. ${ }^{[63]}$ & 2000 & 19 & 19 & $\begin{array}{l}\mathrm{SC}=17 \\
\mathrm{MC}=2 \\
\mathrm{FP}=15\end{array}$ & $15.8 \%$ radiological & 0 & 24 & $\begin{array}{l}15.8 \% \text { conversions } \\
75 \% \text { reflux esophagitis } \\
\text { without FP }\end{array}$ \\
\hline Ponsky et al. ${ }^{[64]}$ & 2003 & 28 & 28 & $F P=28$ & ०\% radiological & 0 & 12 & \\
\hline Diaz et al. ${ }^{[65]}$ & 2003 & 116 & 48 & $\begin{array}{l}S C=110 \\
M C=6 \\
F P=114 \\
E L=6\end{array}$ & $32 \%$ radiological & 1.7 & 30 & $\begin{array}{l}4.3 \% \text { major } \\
\text { complications }\end{array}$ \\
\hline Horstmann et al. ${ }^{[66]}$ & 2004 & 16 & 16 & $\begin{array}{l}M C=16 \\
F P=16\end{array}$ & 0\% radiological & 0 & 14 & $\begin{array}{l}6.25 \% \text { conversions } \\
31 \% \text { pleural injury }\end{array}$ \\
\hline Poncet et al. ${ }^{[67]}$ & 2010 & 89 & 77 & $\begin{array}{l}M C=89 \\
F P=89\end{array}$ & $15.7 \%$ radiological & 0 & 57.5 & $\begin{array}{l}4.4 \% \text { conversions } \\
7.8 \% \text { morbidity }\end{array}$ \\
\hline Daigle et al. ${ }^{[68]}$ & 2015 & 101 & 101 & $\mathrm{SC}=94$ & $\begin{array}{l}16.8 \% \text { endoscopic/ } \\
\text { radiological }\end{array}$ & 0 & 10.9 & $\begin{array}{l}22 \% \text { morbidity } \\
29.7 \% \text { reflux }\end{array}$ \\
\hline Yates et al. ${ }^{[69]}$ & 2015 & $11 \mathrm{HR}$ & 10 & $\mathrm{TG}=11$ & 0\% symptomatic & $\mathrm{N} / \mathrm{A}$ & 3 & $\begin{array}{l}2 \text { readmissions } \\
2 \text { TG dislocations }\end{array}$ \\
\hline Higashi et al. ${ }^{[70]}$ & 2017 & $8 \mathrm{HR}$ & 100 & & ०\% symptomatic & $0 \%$ & 48 & \\
\hline
\end{tabular}

HR: high risk patients; GP: gastropexy; MC: mesh cruroplasty; SC: simple cruroplasty; FP: fundoplication; EL: esophageal lengthening; TG: tube gastrostomy

The rationale for adding a fundoplication is twofold: treating preoperative GER symptoms and preventing the postoperative onset of GER. GER is a frequent clinical manifestation of PEH because the herniation through the diaphragmatic hiatus determines a functional incompetence of the lower esophageal sphincter (LES), favoring the reflux of the gastric contents. GER can also occur "de novo" postoperatively due to altered functional anatomy of the GEJ caused by extensive mediastinal dissection. Furthermore, fundoplication is thought to anchor the cardia below the diaphragm, contributing to the reduction in the rate of recurrences ${ }^{[50]}$. For these reasons, some authors advocate the routine addition of a fundoplication to restore the functional competence of the $\operatorname{LES}^{[71]}$.

Other authors sustain the selective addition of fundoplication during PEH repair depending on the presence of preoperative GER or altered esophageal motility at esophageal manometry. They believe that the intra-abdominal reduction of PEH restores the normal anatomy of the EGJ, therefore no other antireflux operations, with the consequent risk of dysphagia, are needed ${ }^{[72]}$.

However, the LES competence can be difficult to assess preoperatively, because esophageal manometry can be unreliable in the presence of $\mathrm{PEH}^{[73]}$. Furthermore, the incidence of dysphagia following fundoplication is minimal in experienced hands ${ }^{[74]}$.

Müller-Stich et al. ${ }^{[75]}$ performed a RCT comparing mesh-augmented hiatoplasty with or without the addition of a fundoplication. At 12-month follow-up, the fundoplication group had a significantly lower incidence of GER symptoms than hiatoplasty alone, and the subjective results were confirmed by objective upper endoscopy findings. Interestingly, the incidence of gas bloat and dysphagia did not differ between the two groups, leading the authors to favor the systematic addition of an anti-reflux procedure.

In addition, Furnée et al ${ }^{[76]}$ performed a comparative study of patients who underwent PEH repair with or without fundoplication. Of the 20 patients who did not receive fundoplication, new onset of esophagitis occurred in $28 \%$, and pathological acid exposure was demonstrated in $39 \%$. In the fundoplication group, $8.7 \%$ of patients experienced dysphagia. The authors concluded that, since the rate of postoperative side effects of fundoplication is low, while objective evidence of postoperatively de novo onset of GER occurred frequently, the addition of a fundoplication should be recommended during PEH repair. 
To date, there is no consensus on the type of wrap and on the fixation of the fundoplication to the esophagus or the diaphragmatic pillars ${ }^{[28]}$. In a systematic review of the literature, including 24 studies, Andolfi et al. ${ }^{[77]}$ concluded that the preferred approach should be a total fundoplication when the esophageal motility is normal.

\section{CONCLUSION}

The current review of the literature shows that the controversies regarding the optimal repair of paraesophageal hernia, including the best technique for crural closure, the addition of a fundoplication, and of esophageal lengthening procedures, remain unresolved. The wide heterogeneity of techniques and materials, together with the low incidence of $\mathrm{PEH}$, makes it difficult to investigate the specific role of the single technical factors concurring in PEH repair.

\section{DECLARATIONS}

\section{Authors' contributions}

Made substantial contributions to conception and design of the study and performed data analysis and interpretation: Ugliono E, Rebecchi F

Performed data acquisition, as well as provided administrative, technical, and material support: Seno E, Morino M

\section{Availability of data and materials}

Not applicable.

\section{Financial support and sponsorship}

None.

\section{Conflicts of interest}

All authors declared that there are no conflicts of interest.

\section{Ethical approval and consent to participate}

Not applicable.

\section{Consent for publication}

Not applicable.

\section{Copyright}

(c) The Author(s) 2021.

\section{REFERENCES}

1. Kahrilas PJ, Kim HC, Pandolfino JE. Approaches to the diagnosis and grading of hiatal hernia. Best Pract Res Clin Gastroenterol 2008;22:601-16.

2. Hashemi M, Sillin LF, Peters JH. Current concepts in the management of paraesophageal hiatal hernia. J Clin Gastroenterol 1999;29:8-13.

3. Sihvo EI, Salo JA, Räsänen JV, Rantanen TK. Fatal complications of adult paraesophageal hernia: a population-based study. $J$ Thorac Cardiovasc Surg 2009;137:419-24.

4. Mitiek MO, Andrade RS. Giant hiatal hernia. Ann Thorac Surg 2010;89:S2168-73.

5. Kohn GP, Price RR, DeMeester SR, et al; SAGES Guidelines Committee. Guidelines for the management of hiatal hernia. Surg Endosc 2013;27:4409-28.

6. Skinner DB, Belsey RH. Surgical management of esophageal reflux and hiatus hernia. Long-term results with 1,030 patients. $J$ Thorac Cardiovasc Surg 1967;53:33-54.

7. Hill LD. Incarcerated paraesophageal hernia. Am J Surg 1973;126:286-91.

8. Stylopoulos N, Gazelle GS, Rattner DW. Paraesophageal hernias: operation or observation? Ann Surg 2002;236:492-500; discussion 500-1. 
9. Jassim H, Seligman JT, Frelich M, et al. A population-based analysis of emergent versus elective paraesophageal hernia repair using the Nationwide Inpatient Sample. Surg Endosc 2014;28:3473-8.

10. Kaplan JA, Schecter S, Lin MY, Rogers SJ, Carter JT. Morbidity and Mortality Associated With Elective or Emergency Paraesophageal Hernia Repair. JAMA Surg 2015;150:1094-6.

11. Jung JJ, Naimark DM, Behman R, Grantcharov TP. Approach to asymptomatic paraesophageal hernia: watchful waiting or elective laparoscopic hernia repair? Surg Endosc 2018;32:864-71.

12. Morrow EH, Chen J, Patel R, et al. Watchful waiting versus elective repair for asymptomatic and minimally symptomatic paraesophageal hernias: A cost-effectiveness analysis. Am J Surg 2018;216:760-3.

13. Draaisma WA, Gooszen HG, Tournoij E, Broeders IA. Controversies in paraesophageal hernia repair: a review of literature. Surg Endosc 2005;19:1300-8.

14. Cuschieri A, Shimi S, Nathanson LK. Laparoscopic reduction, crural repair, and fundoplication of large hiatal hernia. The American Journal of Surgery 1992;163:425-30.

15. McLaren PJ, Hart KD, Hunter JG, Dolan JP. Paraesophageal Hernia Repair Outcomes Using Minimally Invasive Approaches. JAMA Surg 2017;152:1176-8.

16. Kubasiak J, Hood KC, Daly S, et al. Improved patient outcomes in paraesophageal hernia repair using a laparoscopic approach: a study of the national surgical quality improvement program data. Am Surg 2014;80:884-9.

17. Klinginsmith M, Jolley J, Lomelin D, Krause C, Heiden J, Oleynikov D. Paraesophageal hernia repair in the emergency setting: is laparoscopy with the addition of a fundoplication the new gold standard? Surg Endosc 2016;30:1790-5.

18. Brenkman HJ, Parry K, van Hillegersberg R, Ruurda JP. Robot-Assisted Laparoscopic Hiatal Hernia Repair: Promising Anatomical and Functional Results. J Laparoendosc Adv Surg Tech A 2016;26:465-9.

19. Galvani CA, Loebl H, Osuchukwu O, Samamé J, Apel ME, Ghaderi I. Robotic-Assisted Paraesophageal Hernia Repair: Initial Experience at a Single Institution. J Laparoendosc Adv Surg Tech A 2016;26:290-5.

20. Vasudevan V, Reusche R, Nelson E, Kaza S. Robotic paraesophageal hernia repair: a single-center experience and systematic review. $J$ Robot Surg 2018;12:81-6.

21. O'Rourke RW, Khajanchee YS, Urbach DR, et al. Extended transmediastinal dissection: an alternative to gastroplasty for short esophagus. Arch Surg 2003;138:735-40.

22. Oleynikov D, Jolley JM. Paraesophageal hernia. Surg Clin North Am 2015;95:555-65.

23. Trus TL, Bax T, Richardson WS, et al. Complications of laparoscopic paraesophageal hernia repair. J Gastrointest Surg 1997;1:221-7; discussion 228.

24. Kakarlapudi GV, Awad ZT, Haynatzki G, Sampson T, Stroup G, Filipi CJ. The effect of diaphragmatic stressors on recurrent hiatal hernia. Hernia 2002;6:163-6.

25. Robertson-More C, Prasad S, Gill R, Church N, Mitchell P, Debru E. Early Routine Use of Upper GI Contrast Series Post Paraesophageal Hernia Repair: A Single Institution Consecutive Case Series. Surg Laparosc Endosc Percutan Tech 2019;29:203-6.

26. Lidor AO, Kawaji Q, Stem M, et al. Defining recurrence after paraesophageal hernia repair: correlating symptoms and radiographic findings. Surgery 2013;154:171-8.

27. Rathore MA, Andrabi SI, Bhatti MI, Najfi SMH, McMurray A. Metaanalysis of recurrence after laparoscopic repair of paraesophageal hernia. JSLS 2007;11:456-60.

28. Auyang ED, Pellegrini CA. How I do it: laparoscopic paraesophageal hernia repair. J Gastrointest Surg 2012;16:1406-11.

29. Edye M, Salky B, Posner A, Fierer A. Sac excision is essential to adequate laparoscopic repair of paraesophageal hernia. Surg Endosc 1998;12:1259-63.

30. Greene CL, DeMeester SR, Zehetner J, Worrell SG, Oh DS, Hagen JA. Diaphragmatic relaxing incisions during laparoscopic paraesophageal hernia repair. Surg Endosc 2013;27:4532-8.

31. Targarona EM, Bendahan G, Balague C, Garriga J, Trias M. Mesh in the hiatus: a controversial issue. Arch Surg 2004;139:1286-96; discussion 1296

32. Gordon AC, Gillespie C, Son J, Polhill T, Leibman S, Smith GS. Long-term outcomes of laparoscopic large hiatus hernia repair with nonabsorbable mesh. Dis Esophagus 2018;31.

33. Alicuben ET, Worrell SG, DeMeester SR Resorbable biosynthetic mesh for crural reinforcement during hiatal hernia repair. Am Surg 2014;80:1030-3.

34. Powell BS, Wandrey D, Voeller GR. A technique for placement of a bioabsorbable prosthesis with fibrin glue fixation for reinforcement of the crural closure during hiatal hernia repair. Hernia 2013;17:81-4.

35. Weitzendorfer M, Pfandner R, Antoniou SA, Schwaiger-Hengstschläger C, Emmanuel K, Koch OO. Short-term results after laparoscopic repair of giant hiatal hernias with pledgeted sutures: a retrospective analysis. Hernia 2019;23:397-401.

36. Morino M, Giaccone C, Pellegrino L, Rebecchi F. Laparoscopic management of giant hiatal hernia: factors influencing long-term outcome. Surg Endosc 2006;20:1011-6.

37. Frantzides CT, Madan AK, Carlson MA, Stavropoulos GP. A prospective, randomized trial of laparoscopic polytetrafluoroethylene (PTFE) patch repair vs simple cruroplasty for large hiatal hernia. Arch Surg 2002;137:649-52.

38. Tatum RP, Shalhub S, Oelschlager BK, Pellegrini CA. Complications of PTFE mesh at the diaphragmatic hiatus. $J$ Gastrointest Surg 2008;12:953-7.

39. Oelschlager BK, Pellegrini CA, Hunter J, et al. Biologic prosthesis reduces recurrence after laparoscopic paraesophageal hernia repair: a multicenter, prospective, randomized trial. Ann Surg 2006;244:481-90. 
40. Oelschlager BK, Pellegrini CA, Hunter JG, et al. Biologic prosthesis to prevent recurrence after laparoscopic paraesophageal hernia repair: long-term follow-up from a multicenter, prospective, randomized trial. J Am Coll Surg 2011;213:461-8.

41. Antoniou SA, Müller-Stich BP, Antoniou GA, et al. Laparoscopic augmentation of the diaphragmatic hiatus with biologic mesh versus suture repair: a systematic review and meta-analysis. Langenbecks Arch Surg 2015;400:577-83.

42. Watson DI, Thompson SK, Devitt PG, et al. Laparoscopic repair of very large hiatus hernia with sutures versus absorbable mesh versus nonabsorbable mesh: a randomized controlled trial. Ann Surg 2015;261:282-9.

43. Watson DI, Thompson SK, Devitt PG, et al. Five Year Follow-up of a Randomized Controlled Trial of Laparoscopic Repair of Very Large Hiatus Hernia With Sutures Versus Absorbable Versus Nonabsorbable Mesh. Ann Surg 2020;272:241-7.

44. Memon MA, Siddaiah-Subramanya M, Yunus RM, Memon B, Khan S. Suture Cruroplasty Versus Mesh Hiatal Herniorrhaphy for Large Hiatal Hernias (HHs): An Updated Meta-Analysis and Systematic Review of Randomized Controlled Trials. Surg Laparosc Endosc Percutan Tech 2019;29:221-32.

45. Sathasivam R, Bussa G, Viswanath Y, et al. 'Mesh hiatal hernioplasty' versus 'suture cruroplasty' in laparoscopic para-oesophageal hernia surgery; a systematic review and meta-analysis. Asian J Surg 2019;42:53-60.

46. Tam V, Winger DG, Nason KS. A systematic review and meta-analysis of mesh vs suture cruroplasty in laparoscopic large hiatal hernia repair. Am J Surg 2016;211:226-38.

47. Pallabazzer G, Santi S, Parise P, Solito B, Giusti P, Rossi M. Giant hiatal hernias: direct hiatus closure has an acceptable recurrence rate. Updates Surg 2011;63:75-81.

48. Furtado RV, Vivian SJ, van der Wall H, Falk GL. Medium-term durability of giant hiatus hernia repair without mesh. Ann R Coll Surg Engl 2016;98:450-5.

49. Koetje JH, Oor JE, Roks DJ, Van Westreenen HL, Hazebroek EJ, Nieuwenhuijs VB. Equal patient satisfaction, quality of life and objective recurrence rate after laparoscopic hiatal hernia repair with and without mesh. Surg Endosc 2017;31:3673-80.

50. Furnée EJ, Smith CD, Hazebroek EJ. The Use of Mesh in Laparoscopic Large Hiatal Hernia Repair: A Survey of European Surgeons. Surg Laparosc Endosc Percutan Tech 2015;25:307-11.

51. Pfluke JM, Parker M, Bowers SP, Asbun HJ, Daniel Smith C. Use of mesh for hiatal hernia repair: a survey of SAGES members. Surg Endosc 2012;26:1843-8.

52. Horvath KD, Swanstrom LL, Jobe BA. The short esophagus: pathophysiology, incidence, presentation, and treatment in the era of laparoscopic antireflux surgery. Ann Surg 2000;232:630-40.

53. Madan AK, Frantzides CT, Patsavas KL. The myth of the short esophagus. Surg Endosc 2004;18:31-4.

54. Mattioli S, Lugaresi ML, Costantini M, et al. The short esophagus: intraoperative assessment of esophageal length. $J$ Thorac Cardiovasc Surg 2008;136:834-41.

55. Swanstrom LL, Marcus DR, Galloway GQ. Laparoscopic collis gastroplasty is the treatment of choice for the shortened esophagus. Am J Surg 1996;171:477-81.

56. Johnson AB, Oddsdottir M, Hunter JG. Laparoscopic Collis gastroplasty and Nissen fundoplication. A new technique for the management of esophageal foreshortening. Surg Endosc 1998;12:1055-60.

57. Kunio NR, Dolan JP, Hunter JG. Short esophagus. Surg Clin North Am 2015;95:641-52.

58. Nason KS, Luketich JD, Awais O, et al. Quality of life after collis gastroplasty for short esophagus in patients with paraesophageal hernia. Ann Thorac Surg 2011;92:1854-60; discussion 1860-1.

59. Boerema WJ. Anterior gastropexy: a simple operation for hiatus hernia. Aust N Z J Surg 1969;39:173-5.

60. Davies CJ. A survey of the results of the Boerema anterior gastropexy for hiatus hernia over a 4-year period. Br J Surg 1975;62:19-22.

61. Agwunobi AO, Bancewicz J, Attwood SE. Simple laparoscopic gastropexy as the initial treatment of paraoesophageal hiatal hernia. $\mathrm{Br} J$ Surg 1998;85:604-6.

62. Hawasli A, Zonca S. Laparoscopic repair of paraesophageal hiatal hernia. Am Surg 1998;64:703-10.

63. van der Peet DL, Klinkenberg-Knol EC, Alonso Poza A, Sietses C, Eijsbouts QA, Cuesta MA. Laparoscopic treatment of large paraesophageal hernias: both excision of the sac and gastropexy are imperative for adequate surgical treatment. Surg Endosc 2000;14:1015-8.

64. Ponsky J, Rosen M, Fanning A, Malm J. Anterior gastropexy may reduce the recurrence rate after laparoscopic paraesophageal hernia repair. Surg Endosc 2003;17:1036-41.

65. Diaz S, Brunt LM, Klingensmith ME, Frisella PM, Soper NJ. Laparoscopic paraesophageal hernia repair, a challenging operation: medium-term outcome of 116 patients. J Gastrointest Surg 2003;7:59-67.

66. Horstmann R, Klotz A, Classen C, Palmes D. Feasibility of surgical technique and evaluation of postoperative quality of life after laparoscopic treatment of intrathoracic stomach. Langenbecks Arch Surg 2004;389:23-31.

67. Poncet G, Robert M, Roman S, Boulez JC. Laparoscopic repair of large hiatal hernia without prosthetic reinforcement: late results and relevance of anterior gastropexy. J Gastrointest Surg 2010;14:1910-6.

68. Daigle CR, Funch-Jensen P, Calatayud D, Rask P, Jacobsen B, Grantcharov TP. Laparoscopic repair of paraesophageal hernia with anterior gastropexy: a multicenter study. Surg Endosc 2015;29:1856-61.

69. Yates RB, Hinojosa MW, Wright AS, Pellegrini CA, Oelschlager BK. Laparoscopic gastropexy relieves symptoms of obstructed gastric volvulus in highoperative risk patients. Am J Surg 2015;209:875-80; discussion 880.

70. Higashi S, Nakajima K, Tanaka K, et al. Laparoscopic anterior gastropexy for type III/IV hiatal hernia in elderly patients. Surg Case Rep 2017;3:45.

71. Casabella F, Sinanan M, Horgan S, Pellegrini CA. Systematic use of gastric fundoplication in laparoscopic repair of paraesophageal 
hernias. Am J Surg 1996;171:485-9.

72. Morris-Stiff G, Hassn A. Laparoscopic paraoesophageal hernia repair: fundoplication is not usually indicated. Hernia 2008;12:299-302.

73. Khanna A, Finch G. Paraoesophageal herniation: a review. Surgeon 2011;9:104-11.

74. Marano L, Schettino M, Porfidia R, et al. The laparoscopic hiatoplasty with antireflux surgery is a safe and effective procedure to repair giant hiatal hernia. BMC Surg 2014;14:1.

75. Müller-Stich BP, Achtstätter V, Diener MK, et al. Repair of Paraesophageal Hiatal Hernias - Is a Fundoplication Needed? A Randomized Controlled Pilot Trial. J Am Coll Surg 2015;221:602-10.

76. Furnée EJ, Draaisma WA, Gooszen HG, Hazebroek EJ, Smout AJ, Broeders IA. Tailored or routine addition of an antireflux fundoplication in laparoscopic large hiatal hernia repair: a comparative cohort study. World J Surg 2011;35:78-84.

77. Andolfi C, Plana A, Furno S, Fisichella PM. Paraesophageal Hernia and Reflux Prevention: Is One Fundoplication Better than the Other? World J Surg 2017;41:2573-82. 